\title{
Active Noise Cancellation for Ventilation Ducts Using a Pair of Loudspeakers by Sampled-Data $\mathscr{H}_{\infty}$ Optimization
}

\author{
Yasuhide Kobayashi ${ }^{1}$ and Hisaya Fujioka ${ }^{2}$ \\ ${ }^{1}$ Department of Mechanical Engineering, Faculty of Engineering, Nagaoka University of Technology, Nagaoka, Niigata 940-2188, Japan \\ ${ }^{2}$ Department of Applied Analysis and Complex Dynamical Systems, Graduate School of Informatics, Kyoto University, \\ Kyoto 606-8501, Japan
}

Correspondence should be addressed to Yasuhide Kobayashi, kobayasi@vos.nagaokaut.ac.jp

Received 2 December 2007; Accepted 14 March 2008

Recommended by Marek Pawelczyk

We deal with the design of active noise control systems for ventilation ducts with a pair of loud-speakers based on sampleddata $\mathscr{H}_{\infty}$ optimization, in order to improve our previous study with a single loudspeaker. The resultant controller requires lower computational complexity for the implementation, compared to the standard time-varying adaptive controllers, since it is time-invariant and the order is comparable. Moreover, the effect of Swinbanks' source is automatically recovered by the $\mathscr{H}_{\infty}$ optimization. The benefit of the proposed method is also confirmed from experimental results for a real house, where the achieved noise attenuation level is about twice as much as that of the single loudspeaker case.

Copyright (C) 2008 Y. Kobayashi and H. Fujioka. This is an open access article distributed under the Creative Commons Attribution License, which permits unrestricted use, distribution, and reproduction in any medium, provided the original work is properly cited.

\section{INTRODUCTION}

Active noise cancellation (ANC) is to attenuate noise by the same sound but opposite phase. This technique has been practically used in air conditioning systems in largescale buildings, aircraft cabins, and so on [1]. However, there are many fields such as ventilation ducts for small buildings where ANC is desired but has not been applied yet because the conventional adaptive algorithms for ANC require expensive implementation.

In the previous study [2], by assuming that temperature variation is small as in recent energy-efficient houses so that time-invariant controller can achieve sufficient performance, two types of time-invariant low-order controllers are compared: one is based on the conventional adaptive filter (the filtered-U recursive LMS algorithm) but the coefficients are fixed by the stationary values. Another is obtained by a robust control method (sampled-data $\mathcal{H}_{\infty}$ control). It has been shown that robust control design has advantage to implement inexpensive ANC system for ventilation ducts, because it gives a systematical way to design a controller which is robust against not only plant dynamics variation but also aliasing components of noise. However, further improvement of the sound attenuation is desired.
The method originally proposed by Swinbanks [3] is well known as an effective one for the improvement of the system performance, where an additional loudspeaker is attached to cancel out the upstream sound generated by a control source [4]. The method has been examined in detail under adaptive control setup [5]. However, the effect of Swinbanks' source under robust control setup has not been studied. Moreover, experimental results applied to actual ventilation systems installed in houses have not been reported.

In this paper, we examine robust control design of active noise control systems with a pair of loudspeakers in order to improve the system performance. By regarding the loudspeakers as two independent sources, a single-inputmultiple-output (SIMO) controller is also designed to be compared with Swinbanks' source. The validity of robust control design is shown by experimental results using a ventilation duct installed in a real house.

It would be worth mentioning that there have been many studies that deal with the design problem for duct active noise control systems not only in the adaptive control framework $[1,6]$ but also in the robust control framework [7-10] under the simple duct setup, while the comparison of the adaptive and robust control with a real ventilation system has been firstly reported in [2]. Furthermore, robust 


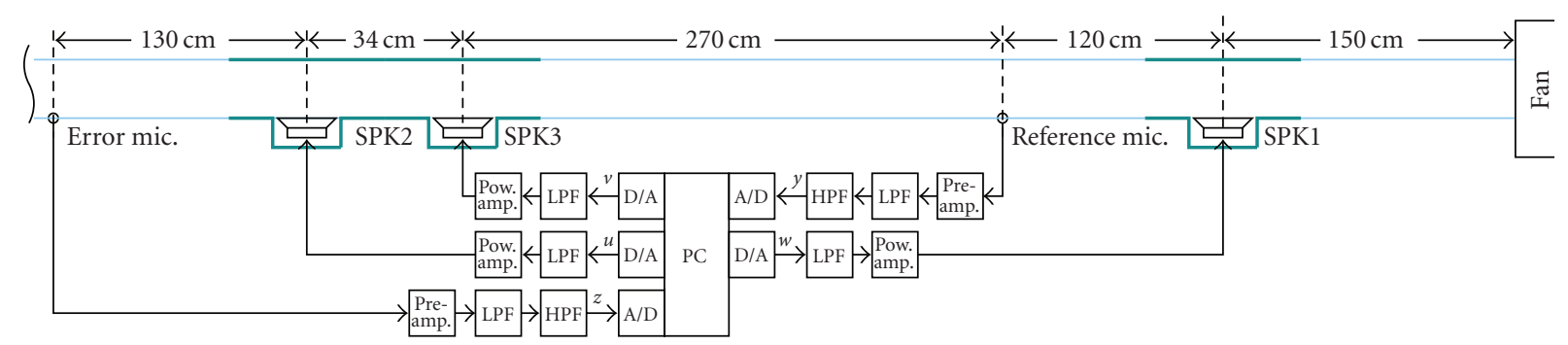

Figure 1: Experimental apparatus.

TABLE 1: Experimental instruments.

\begin{tabular}{ll}
\hline Ventilation fan & Kaneka corp. SV-200U $\left(250 \mathrm{~m}^{3} / \mathrm{h}\right.$, energy-recovery ventilation $)$ \\
\hline Loudspeaker (SPK1) & FOSTEX FW208N woofer speaker with wooden box enclosure \\
Loudspeaker (SPK2 \& SPK3) & FOSTEX FW108N woofer speaker with PVC pipe enclosure \\
Microphones & Electlet condenser type \\
Sound-level meter & RION NL-20 \\
Power amplifier & TOSHIBA TA8213K \\
High-pass filter & NF ELECTRONIC INSTRUMENTS FV-664 $(2 \mathrm{ch}, 80 \mathrm{~Hz}, 24 \mathrm{~dB} / \mathrm{oct})$ \\
Low-pass filter & $500 \mathrm{~Hz} 4$ th-order Butterworth \\
PC & Dell Dimension 2200 (RT-Linux 3.2, kernel 2.4 .22$)$ \\
A/D, D/A & CONTEC AD12-16(PCI), DA12-4(PCI $)(12 \mathrm{bit}, \pm 5 \mathrm{~V}, 10 \mu \mathrm{s})$ \\
\hline
\end{tabular}

control system with Swinbanks' source has not been studied yet.

\section{PROBLEM SETUP}

Figure 1 and Table 1 show the block diagram and instalments of the experimental apparatus which are the same in [2] except that SPK3 and the corresponding D/A channel are attached so that Swinbanks' source is composed of the pair of loudspeakers, SPK2 and SPK3.

In addition, for simplicity of robust control design in this paper, SPK1 is used as a noise source in the modeling stage of robust control design to examine frequency response of the plant model, while in [2] actual fan noise is used.

Figure 2 shows the configuration of the ventilation system installed in a two-stored real house which is also the same as in [2]. The grilles are attached to the ceiling of each floor, and the ANC system is connected between fresh-air grilles and the ventilation fan.

In this paper, we examine the following cases to derive the control sources SPK2 and SPK3.

Case (a) - a single loudspeaker-by setting $v(t)=0$, only SPK2 is used to generate control sound.

Case (b)—swinbanks' source [5]—by setting

$$
v(t)=-u(t-\tau), \quad \tau=\frac{d}{c_{0}},
$$

SPK3 is driven to cancel out the upstream sound generated by SPK2, where $d$ is the distance between SPK2 and SPK3, and $c_{0}$ is the sound speed.
Case (c) — an array of two loudspeakers - by setting $v(t)$ free to $u(t), \mathrm{SPK} 2$ and SPK3 are driven as independent sources.

Our primal motivation is to improve the performance by using a pair of loudspeakers (cases (b) and (c)) instead of a single loudspeaker (case (a)) in the robust control framework. In Swinbanks' source, the upstream loudspeaker is driven by a downstream one in an ad hoc way by (1). Hence our second motivation is to further improve the performance by giving more freedom to the upstream loudspeaker in case (c), although it has been reported in adaptive control framework that the performance is not improved if we replace the delay in case (b) by an adaptive filter to enlarge the design freedom [5].

In the experiments for case (b) below, (1) is approximately implemented as a real-time module of RTLinux that updates the signal $v(t)$ at every 0.1 millisecond which is considered to be short enough to avoid aliasing effect. In addition, by letting $d=0.34 \mathrm{~m}$ from Figure 1 and $c_{0}=344 \mathrm{~m} / \mathrm{s}$ from normal temperature environment, we use $\tau=1$ millisecond which exactly corresponds to 10 times of the period of the real-time module mentioned above. Moreover, the cutoff frequency of HPF is determined by considering the frequency range of Swinbanks' source given as $\left[f_{0}, 5 f_{0}\right]$, where $f_{0}:=d / 12 c_{0}$ [5]. The sampling period of controller is 1 millisecond throughout this paper.

\section{ROBUST CONTROL DESIGN}

The design procedure for case (a) is the same as in [10]. The detail of the design procedure for case (c) is omitted but it is 


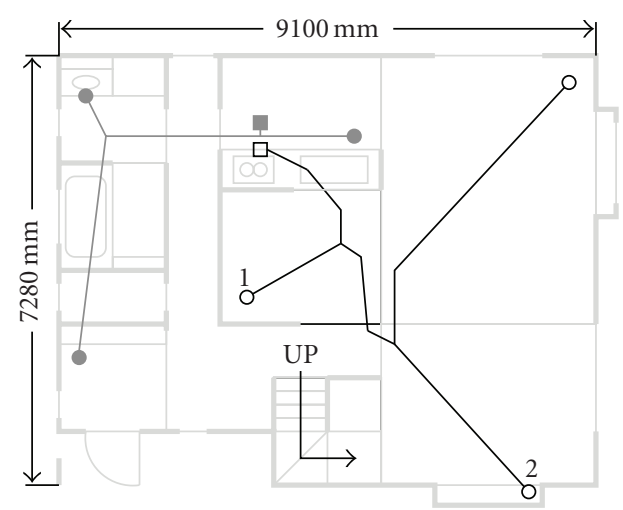

(a) 1 st floor
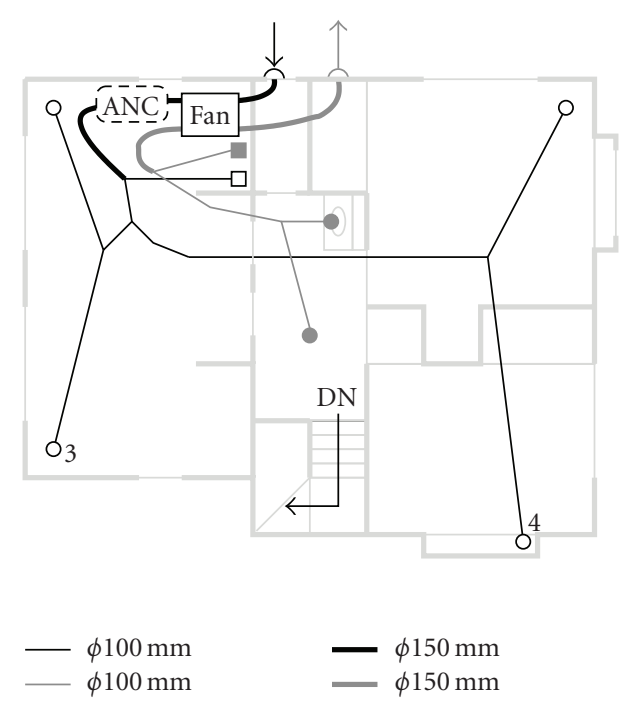

(b) 2nd floor

FIgURE 2: Ventilation system configuration.

done by simply replacing the signal $\left[\begin{array}{ll}u & v\end{array}\right]^{T}$ to $u$ to apply the design process of case (a).

\subsection{Modeling}

The plant models for Figure 1 are determined by frequency response experiment. The system from $\left[\begin{array}{ll}w & u\end{array}\right]^{T}$ to $\left[\begin{array}{ll}z & y\end{array}\right]^{T}$ is considered as the plant transfer function $G(s)$ for cases (a) and (b) as

$$
G(s):=\left[\begin{array}{ll}
G_{z w}(s) & G_{z u}(s) \\
G_{y w}(s) & G_{y u}(s)
\end{array}\right],
$$

while for case (c) $G(s)$ is defined by

$$
G(s):=\left[\begin{array}{lll}
G_{z w}(s) & G_{z u}(s) & G_{z v}(s) \\
G_{y w}(s) & G_{y u}(s) & G_{y v}(s)
\end{array}\right],
$$

where $G_{a b}(s)$ means the transfer function from the signal $b$ to the signal $a$.

Figure 3 shows the frequency response of $G(s)$ and corresponding nominal plant obtained by subspace-based method where the order is 85 . In the figures for $G_{z u}(s)$ and $G_{y u}(s)$, two frequency response results are shown in blue and yellow curves where the former corresponds to cases (a) and (c), while the latter corresponds to case (b). On the other hand, the frequency responses for $G_{z w}(s)$ and $G_{y w}(s)$ shown in blue curves are commonly used to determine nominal plant for all the cases.

In experimental results of case (a), the phase lag becomes larger in the order of $G_{y w}, G_{z u}, G_{y u}$, and $G_{z w}$, of which order coincides with that of the distance from corresponding microphone to speaker. In case (b), remarkable change on $G_{y u}(s)$ is observed compared with case (a): the gain is smaller in the whole frequency range, and the phase lag becomes larger, which can be considered as the result that the distance for sound traveling from the control source to reference microphone becomes larger. This implies that the separation of control input and measured output is improved so that the better performance is expected [11]. Although such remarkable change is not observed in $G_{z u}(s)$, the gain is slightly larger in the middle frequency range of Swinbanks' source, which is the nature of the source reported in [5].

In addition, in order to guarantee the closed-loop system stability against the modeling error of the nominal plant, additive uncertainty model is introduced for feedback-path transfer function. That is, for cases (a) and (b), $G_{y u}(s)$ is considered as

$$
G_{y u}(s)=\bar{G}_{y u}(s)+W(s) \delta(s),
$$

where $\bar{G}_{y u}(s)$ is the nominal plant for $G_{y u}(s), \delta(s)$ is normalized modeling error whose $\mathcal{H}_{\infty}$ norm is less than or equal to 1 , and $W(s)$ is a weighting function which is determined to cover the modeling error as shown in Figure 4. Similarly, for case (c) we define

$$
\left[G_{y u}(s) G_{y v}(s)\right]=\left[\begin{array}{ll}
\bar{G}_{y u}(s) & \bar{G}_{y v}(s)
\end{array}\right]+W(s) \Delta(s),
$$

where $\Delta(s)$ is $1 \times 2$ normalized modeling error. Note that the common weighting function is used for all cases as

$$
W(s)=\frac{0.015\left(s^{2}+2 \zeta_{1} \omega_{1} s+\omega_{1}^{2}\right) \omega_{2}^{2} \omega_{3}^{2}}{\omega_{1}^{2}\left(s^{2}+2 \zeta_{2} \omega_{2} s+\omega_{2}^{2}\right)\left(s^{2}+2 \zeta_{3} \omega_{3} s+\omega_{3}^{2}\right)},
$$

where $\omega_{1}=200, \zeta_{1}=0.9, \omega_{2}=650, \zeta_{2}=0.7, \omega_{3}=2200$, $\zeta_{3}=0.6$.

\subsection{Controller design}

According to the preparation above, sampled-data $\mathscr{H}_{\infty}$ control synthesis [12] is applied to the following digital controller design problem: find a discrete-time controller $K_{d}(z)$ which maximizes positive scalar $\alpha$ so that the following conditions hold:

(i) the closed-loop system of Figure 5 is internally stable;

(ii) there exists positive scalar $d$ such that $\mathscr{L}_{2}$ induced norm of the closed-loop system is less than 1 , 


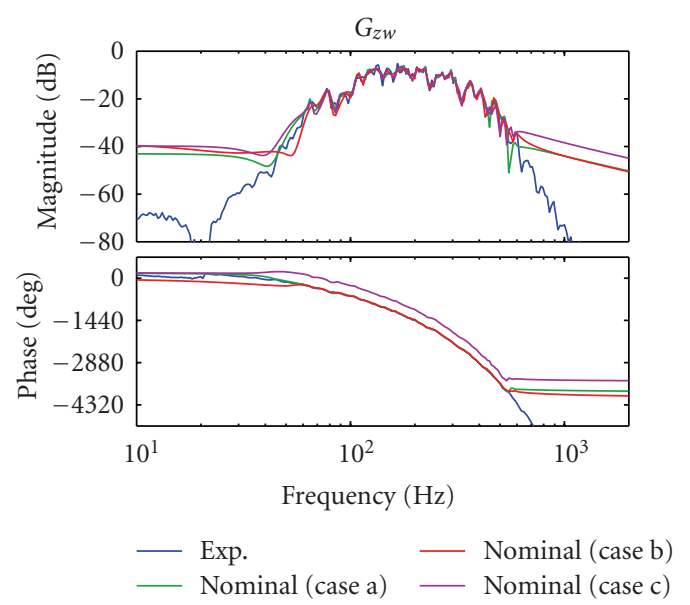

(a) $G_{z w}$

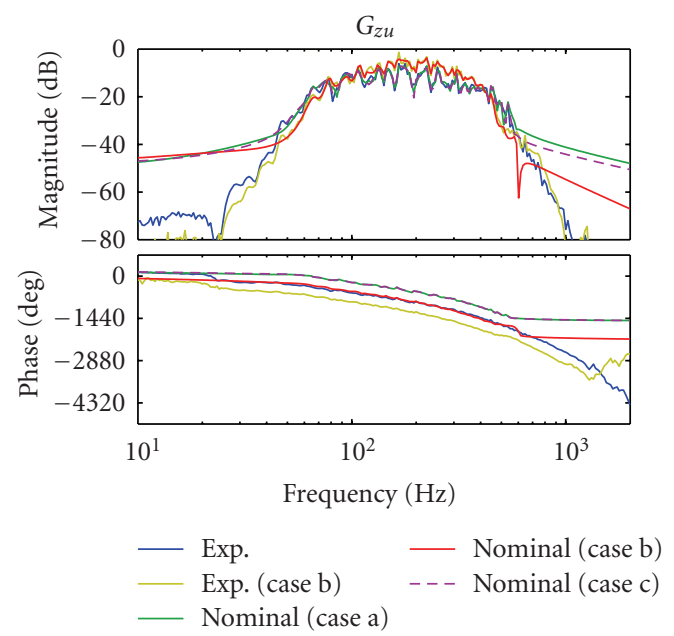

(c) $G_{z v}$

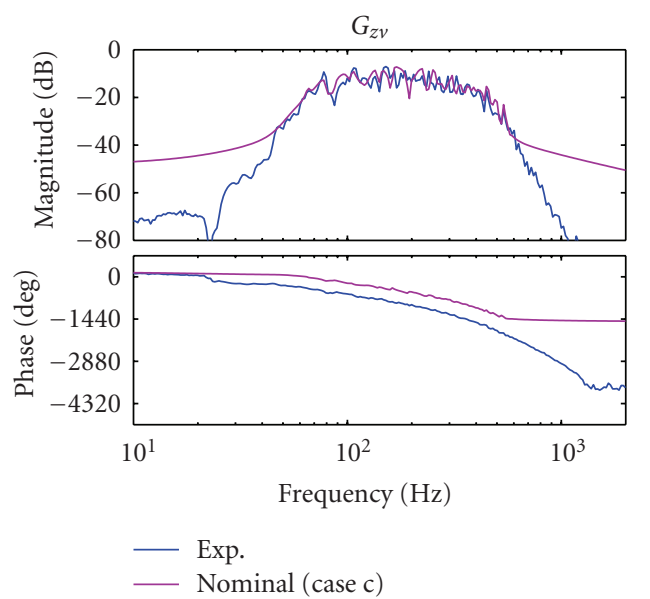

(e) $G_{y u}$

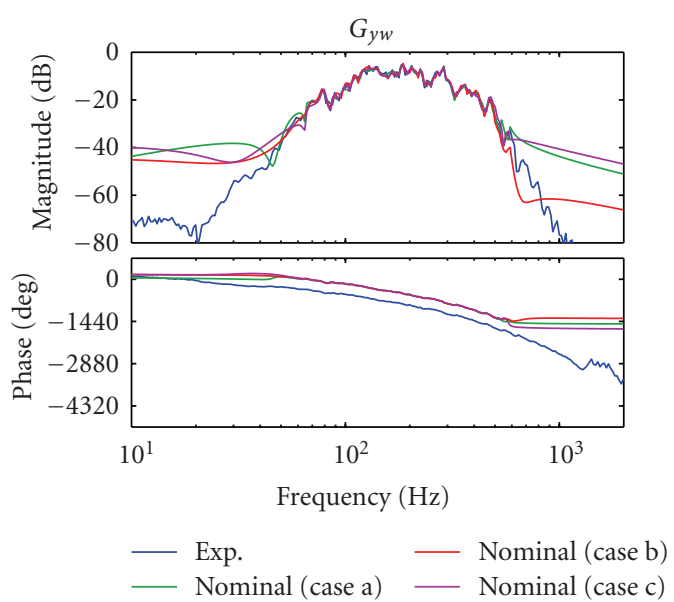

(b) $G_{z u}$

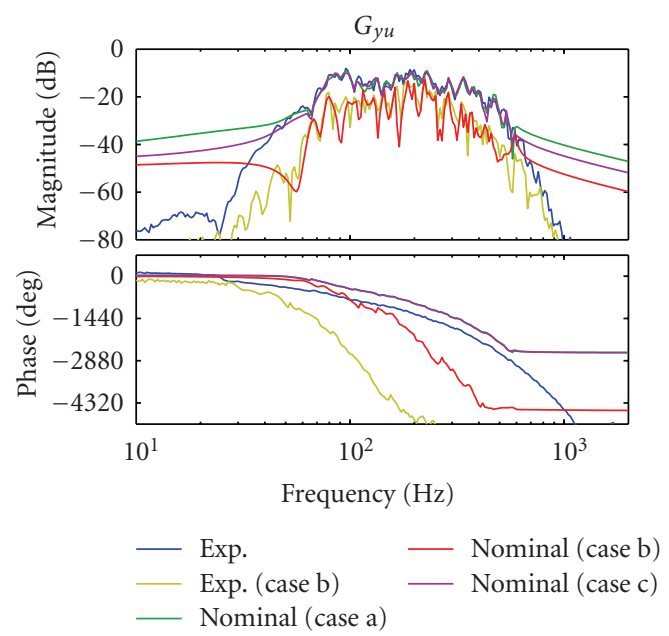

(d) $G_{y w}$

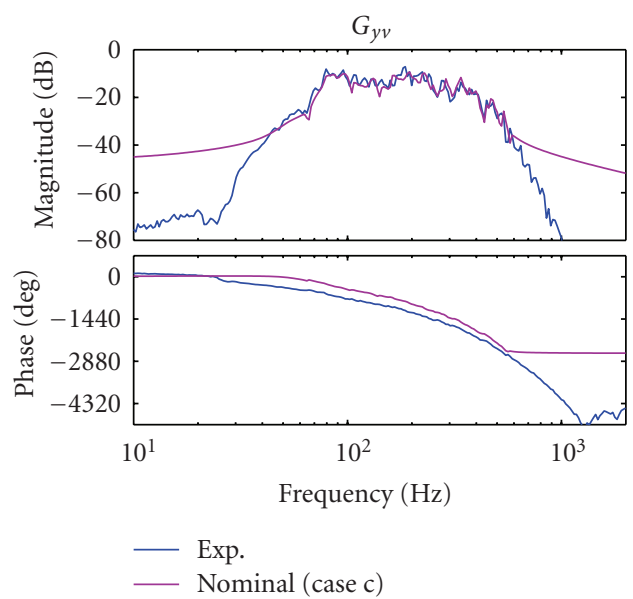

(f) $G_{y v}$

FIGURE 3: Frequency response of plant. 


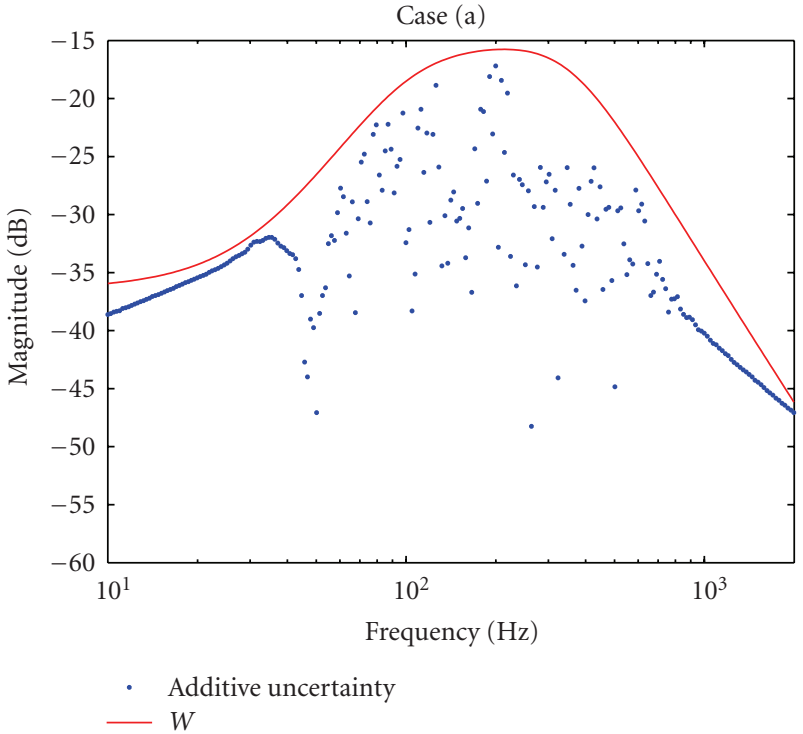

(a)

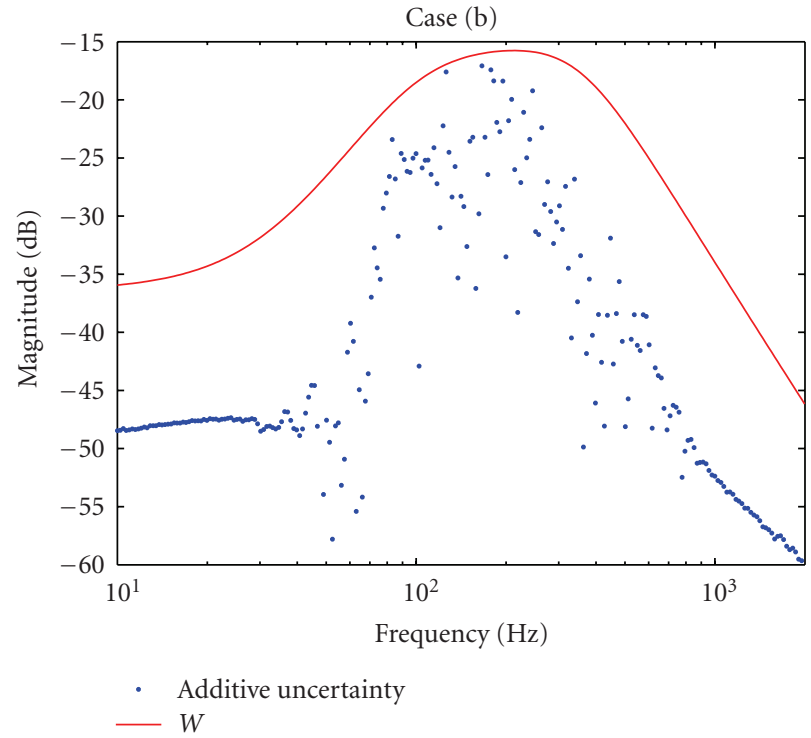

(b)

FIGURE 4: Additive uncertainty and weight.

TABLE 2: Sound pressure level at each grille.

\begin{tabular}{lcccc}
\hline Grille & & \multicolumn{2}{c}{ Sound pressure level $\left(L_{\text {Aeq, } 10 \mathrm{~s}}\right)[\mathrm{dB}]$} \\
& Without control & Case $(\mathrm{a})$ & $\begin{array}{c}\text { With control } \\
\text { Case }(\mathrm{b})\end{array}$ & Case $(\mathrm{c})$ \\
\hline No. 1 & 34.2 & $33.0(-1.2)$ & $32.5(-1.7)$ & $32.1(-2.1)$ \\
No. 2 & 40.4 & $38.8(-1.6)$ & $37.4(-3.0)$ & $37.3(-3.1)$ \\
No. 3 & 31.9 & $30.5(-1.4)$ & $28.9(-3.0)$ & $37.2(-2.7)$ \\
No. 4 & 41.2 & $39.1(-2.1)$ & $37.1)$ & $3(-4.1)$ \\
\hline
\end{tabular}

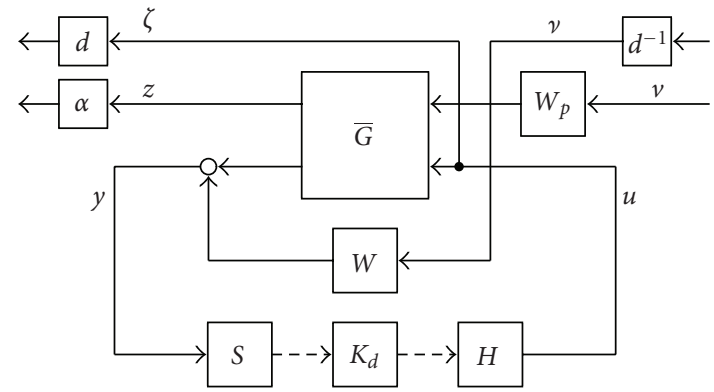

FIGURE 5: Robust performance problem with scalings.

where $S$ is the sampler with sampling period $h=1$ millisecond, $H$ is the zeroth order hold, and $W_{p}(s)$ is a bandpass filter given by

$$
\begin{aligned}
& W_{p}(s)=\left(\frac{s}{s+\omega_{p_{1}}}\right)^{2}\left(\frac{\omega_{p_{2}}}{s+\omega_{p_{2}}}\right)^{2}, \\
& \omega_{p_{1}}=2 \pi \times 80, \quad \omega_{p_{2}}=2 \pi \times 400 .
\end{aligned}
$$

Note that the closed-loop system gain is robustly minimized by maximizing $\alpha$ to improve control performance to attenuate fan noise.
The design results are as follows. The maximal $\alpha=4.64$ was achieved for $d=1.07$ for case (a), and the maximal $\alpha=5.87$ was achieved for $d=1.56$ for case (b), which implies that the closed-loop performance will be improved by Swinbanks' source. Furthermore, $\alpha$ was further improved by case (c) as $\alpha=6.10$ for $d=1.23$, because of the less conservative design. The order of $K_{d}(z)$ is 93 .

\section{COMPARISON OF CONTROLLERS}

In this section, both adaptive and robust controllers are examined, where each adaptive controller for cases (a) and (b) is determined as a fixed 100th IIR filter by using the filtered-U RLMS method (see [2] for design process in detail), while the controller for case (c) is determined as a single-input-double-output fixed 100th IIR filter by using the multiple-channel filtered-U RLMS method [6].

Figure 6 shows adaptive and robust controllers for cases (a) and (b). It can be seen that for the adaptive controllers, the peak gains at about 60,90 , and $100 \mathrm{~Hz}$ become smaller when Swinbanks' source is used, while the similar phenomenon has been reported in [5]. The effect of Swinbanks' source is also observed for the robust controllers shown as the flat gain characteristic within the frequency range from 


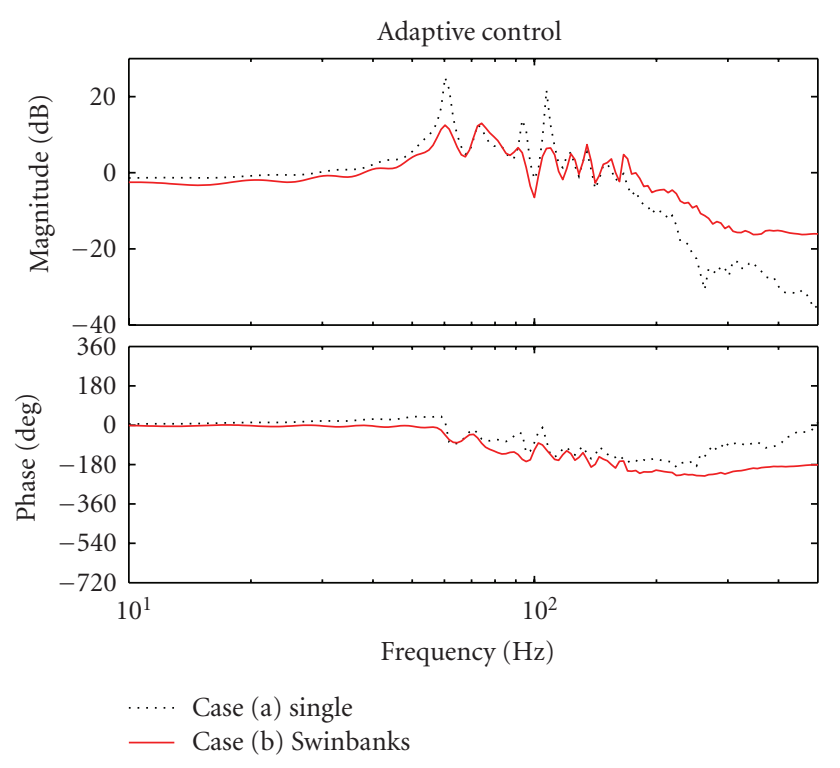

(a) Adaptive control

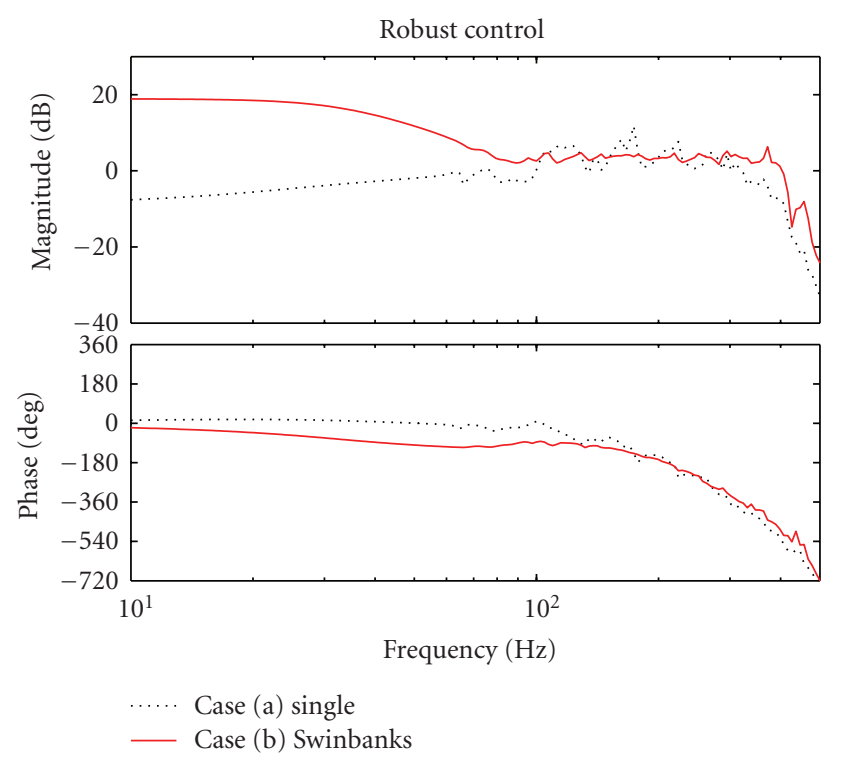

(b) Robust control

Figure 6: Effect of Swinbanks' source.

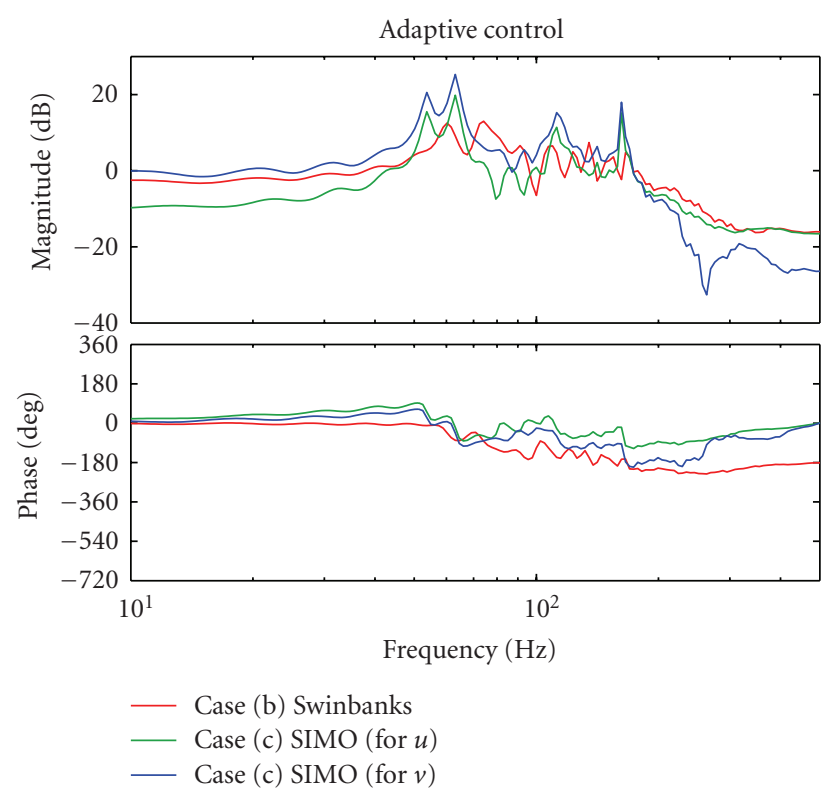

(a) Adaptive control

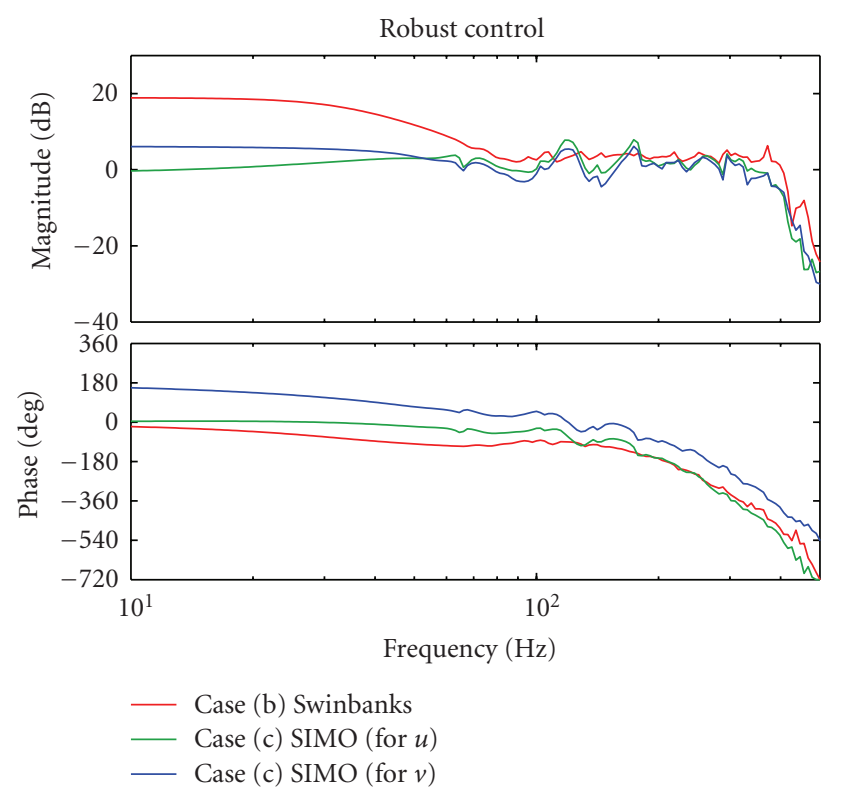

(b) Robust control

Figure 7: Effect of SIMO design.

80 to $400 \mathrm{~Hz}$, while for the case with a single loudspeaker, relatively large peak at about $180 \mathrm{~Hz}$ is appeared.

Figure 7 shows adaptive and robust controllers for cases (b) and (c). It can be seen for the robust controller of case (c) that Swinbanks' source characteristic is automatically obtained, since (i) the gain characteristics of both channels of the controller are similar and (ii) the phase difference is around $180 \mathrm{deg}$. Note that this fact is firstly reported in this paper. Furthermore, by examining the result in detail, advantages of robust control design with case (c) are observed. Firstly, from the gain characteristic, the controller for case (c) has relatively large peaks at about 120 and $170 \mathrm{~Hz}$ compared with case (b), which suggests that the flat gain characteristic of Swinbanks' source is not essential for performance improvement for actual ventilation system. Secondary, in the frequency range around $60 \sim 400$, the gain from $y$ to $v$ is slightly smaller than from $y$ to $u$, which can be interpreted as the result of robust control design to compensate the attenuation of sound due to propagation. In contrast, it might be difficult to give such a reasonable interpretation for the adaptive controller of case (c). 


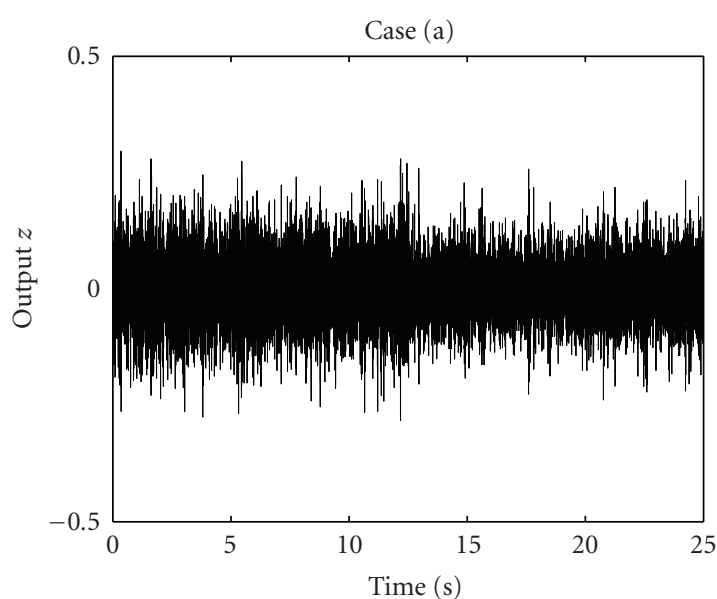

(a)

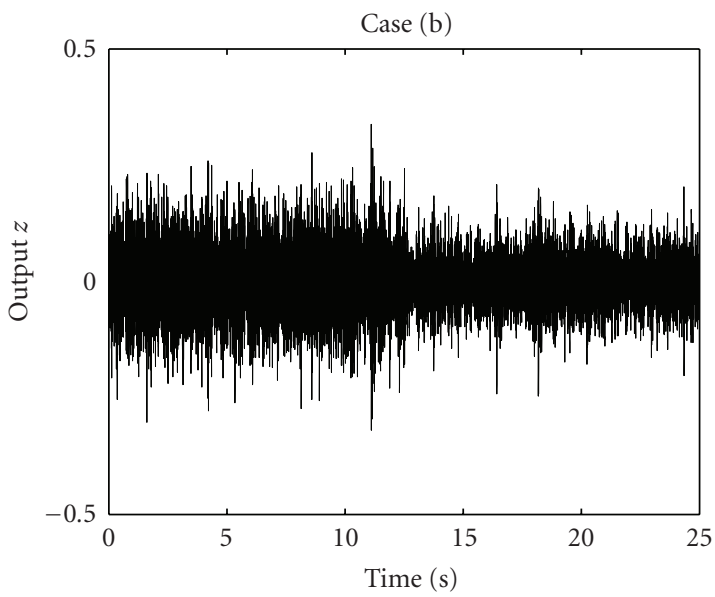

(b)

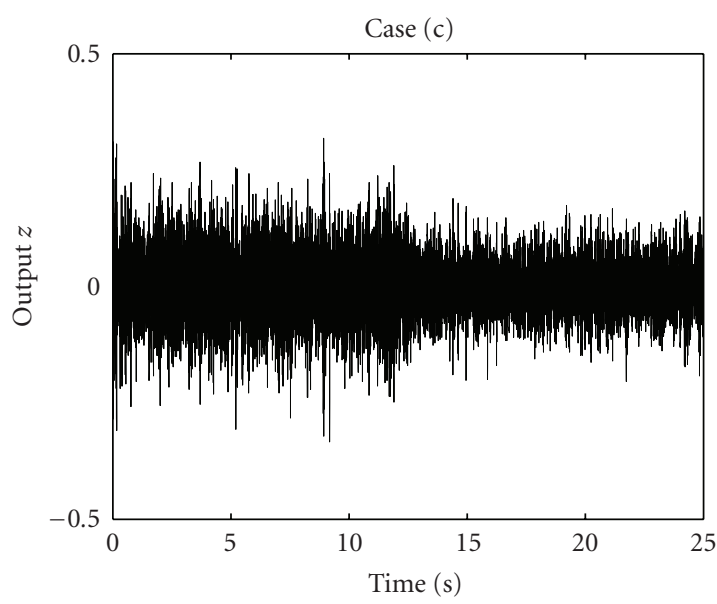

(c)

FIgURE 8: Time response of $z$.

\section{CONTROL EXPERIMENTS}

In this section, robust controllers designed so far are compared by control experiments.

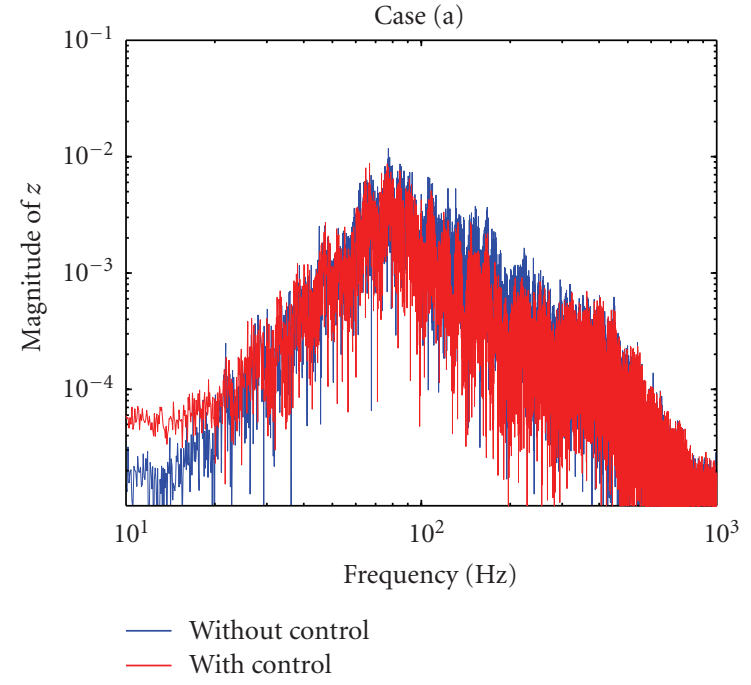

(a)

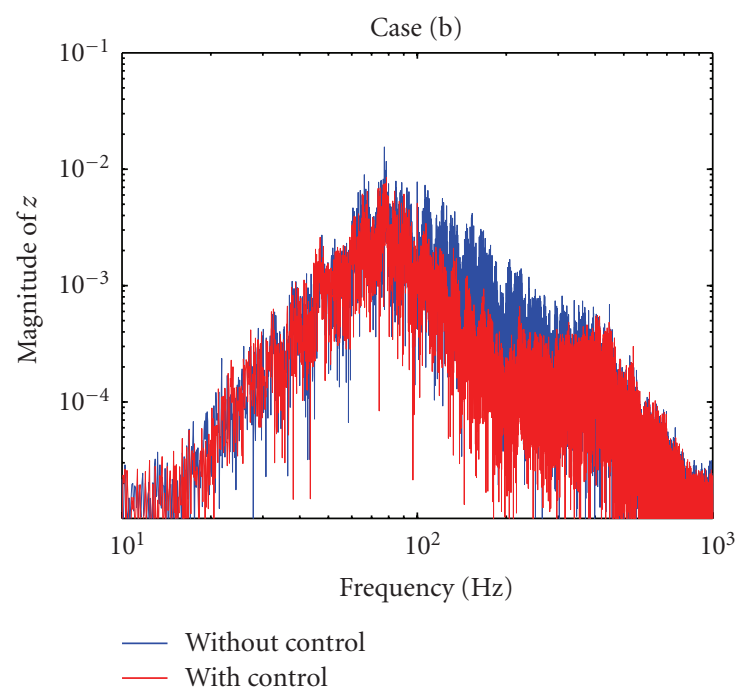

(b)

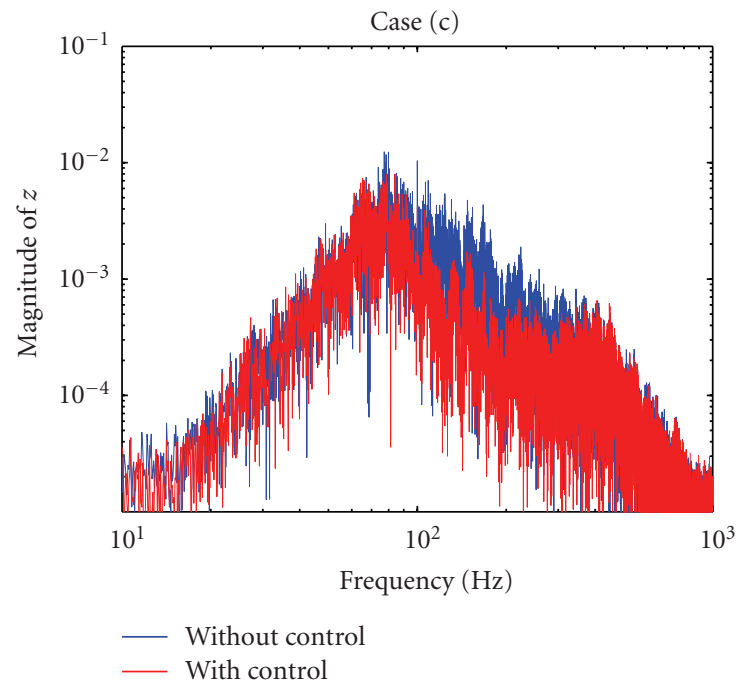

(c)

FIGURE 9: FFT analysis result of $z$. 
Figure 8 shows time response of error microphone signal $z$, where the first 12.5 seconds are without control and the following 12.5 seconds are with control. The smaller sampling period ( 0.5 millisecond) is used for measurement to observe intersample behavior within the sampling period of the controller. It can be seen that cases (b) and (c) show better performance than case (a), while in [5] it has been reported by experimental result that the performances of cases (a) and (b) are similar. This might be caused since directional microphones are used in [5] but not used in this paper.

Figure 9 shows the FFT analysis result of Figure 8. It can be seen that the amplitude of $z$ is reduced within 80 to $400 \mathrm{~Hz}$.

It should be noted that the main frequency component of noise occurs around $100 \mathrm{~Hz}$ whose noise shape differs very much from the open-loop frequency response of $G_{z w}$ shown in Figure 3. Therefore, it is expected that the system performance will be improved by setting the weight function $W_{p}(s)$ to consider the noise shape.

Table 2 shows sound pressure level measured below each grille. It can be seen that the attenuation level of case (b) is about twice the level of case (a), which shows the availability of robust control design for Swinbanks' source. On the other hand, the advantage of case (c) could not be shown (i.e., the attenuation level is similar to case (b)). It is not what we expect from the design results with larger $\alpha$. The reason of this phenomenon is currently under study. One may pose a conjecture that it is from the modeling error. We have considered the modeling error from the control input to the measured output, however, other modeling errors are not reflected in the design. Especially the error of the transfer function from $v$ to $z$ could greatly affect the experimental result of case (c). Indeed, the gain characteristic in Figure 3 shows that there is a relatively large difference between the experimental result and the nominal plant compared with the other transfer functions. Hence we are working on the improvement of the modeling for utilizing the potential advantage of the design setup in case (c), by accounting the modeling error and/or by setting the nominal plant order higher in control design. We could report the results in the future.

\section{CONCLUSIONS}

In this paper, we have examined a robust control design of active noise control systems with a pair of loudspeakers, and the validity of the design method has been shown experimentally by using a ventilation system installed in a real house. The results are summarized as follows.

(i) Firstly, by using Swinbanks' source as a pair of loudspeakers, it has been confirmed that the controller designed by robust control method has flat gain characteristic which has been reported in adaptive control literature as an advantage of using Swinbanks' source.

(ii) Secondly, by considering the pair of loudspeakers as two independent actuators, it has been shown that the controller designed by robust control method is more acceptable than the one designed by adaptive control method, since the similar effect of Swinbanks' source was automatically obtained by the former design but not by the latter one.

(iii) Finally, by comparing the resultant noise attenuation levels of robust controllers with both a single loudspeaker and a pair of loudspeakers, the improvement has been shown by the latter case (i.e., the attenuation level was up to $4 \mathrm{~dB}$ while it was up to $2 \mathrm{~dB}$ for the former case).

Therefore, we conclude together with the result of [2] that the robust control design is useful to implement inexpensive active noise control systems with a pair of loudspeakers for ventilation ducts.

\section{REFERENCES}

[1] S. J. Elliott, Signal Processing for Active Control, Volume in the Signal Processing and Its Applications Series, Academic Press, New York, NY, USA, 2000.

[2] Y. Kobayashi and H. Fujioka, "Inexpensive implementation of active noise control systems for one-dimensional duct with application to a ventilating system," in Proceedings of the 13th International Congress on Sound and Vibration (ICSV '06), Vienna, Austria, July 2006.

[3] M. A. Swinbanks, "The active control of sound propagating in long ducts," Journal of Sound and Vibration, vol. 27, pp. 411436, 1973.

[4] S. Kijimoto, H. Tanaka, Y. Kanemitsu, and K. Matsuda, "Howling cancellation for active noise control with two sound sources," Transactions of the Japan Society of Mechanical Engineers, Series C, vol. 67, no. 656, pp. 52-57, 2001, (Japanese).

[5] J. Winkler and S. J. Elliott, "Adaptive control of broadband sound in ducts using a pair of loudspeakers," Acustica, vol. 81, no. 5, pp. 475-488, 1995.

[6] S. M. Kuo and D. R. Morgan, Active Noise Control Systems: Algorithms and DSP Implementations, John Wiley \& Sons, New York, NY, USA, 1996.

[7] I. R. Petersen, H. R. Pota, and M. R. S. Jahromi, "System identification, uncertainty modelling and actuator placement in the robust control of an acoustic duct," in Proceedings of International Conference on Information, Decision and Control, pp. 41-46, Adelaide, Australia, February 2002.

[8] R. S. Erwin and D. S. Bernstein, "Discrete-time $H_{2} / H_{\infty}$ control of an acoustic duct: $\delta$-domain design and experimental results," in Proceedings of the 36th IEEE Conference on Decision and Control (CDC '97), vol. 1, pp. 281-282, San Diego, Calif, USA, December 1997.

[9] Q.-F. Fan, K. Nonami, and M. Nakano, "Active noise control of one-dimensional exhaust duct using $H^{\infty}$ control theory," Transactions of the Japan Society of Mechanical Engineers, Series C, vol. 62, no. 597, pp. 1805-1810, 1996.

[10] Y. Kobayashi and H. Fujioka, "Active noise control of onedimensional duct via sampled-data $\mathscr{H}_{\infty}$ control," in Proceedings of the 42nd IEEE Conference on Decision and Control (CDC '03), vol. 4, pp. 3900-3904, Maui, Hawaii, USA, December 2003.

[11] D. S. Bernstein, "What makes some control problems hard?" IEEE Control Systems Magazine, vol. 22, no. 4, pp. 8-19, 2002.

[12] T. Chen and B. Francis, Optimal Sampled-Data Control Systems, Springer, New York, NY, USA, 1996. 

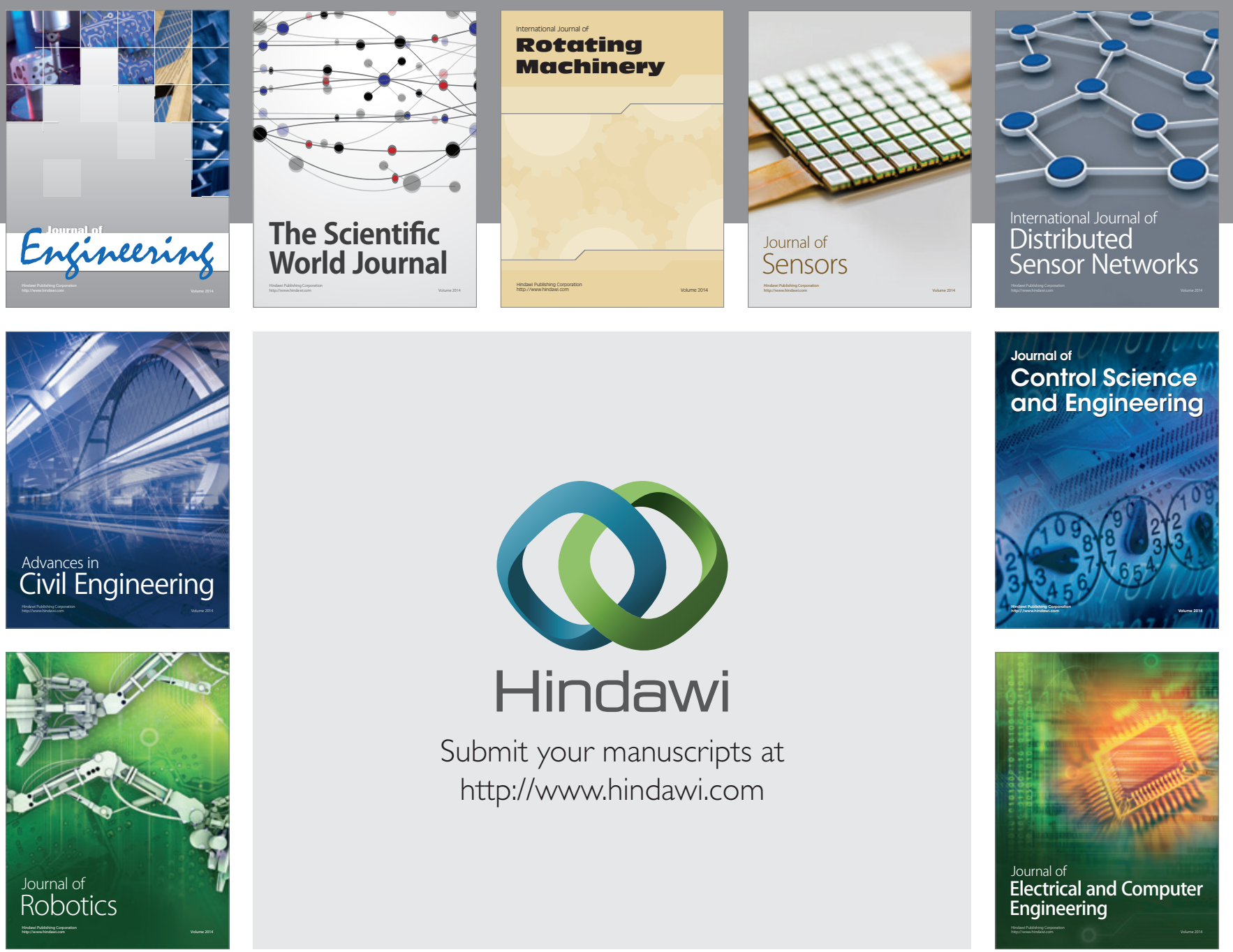

Submit your manuscripts at

http://www.hindawi.com
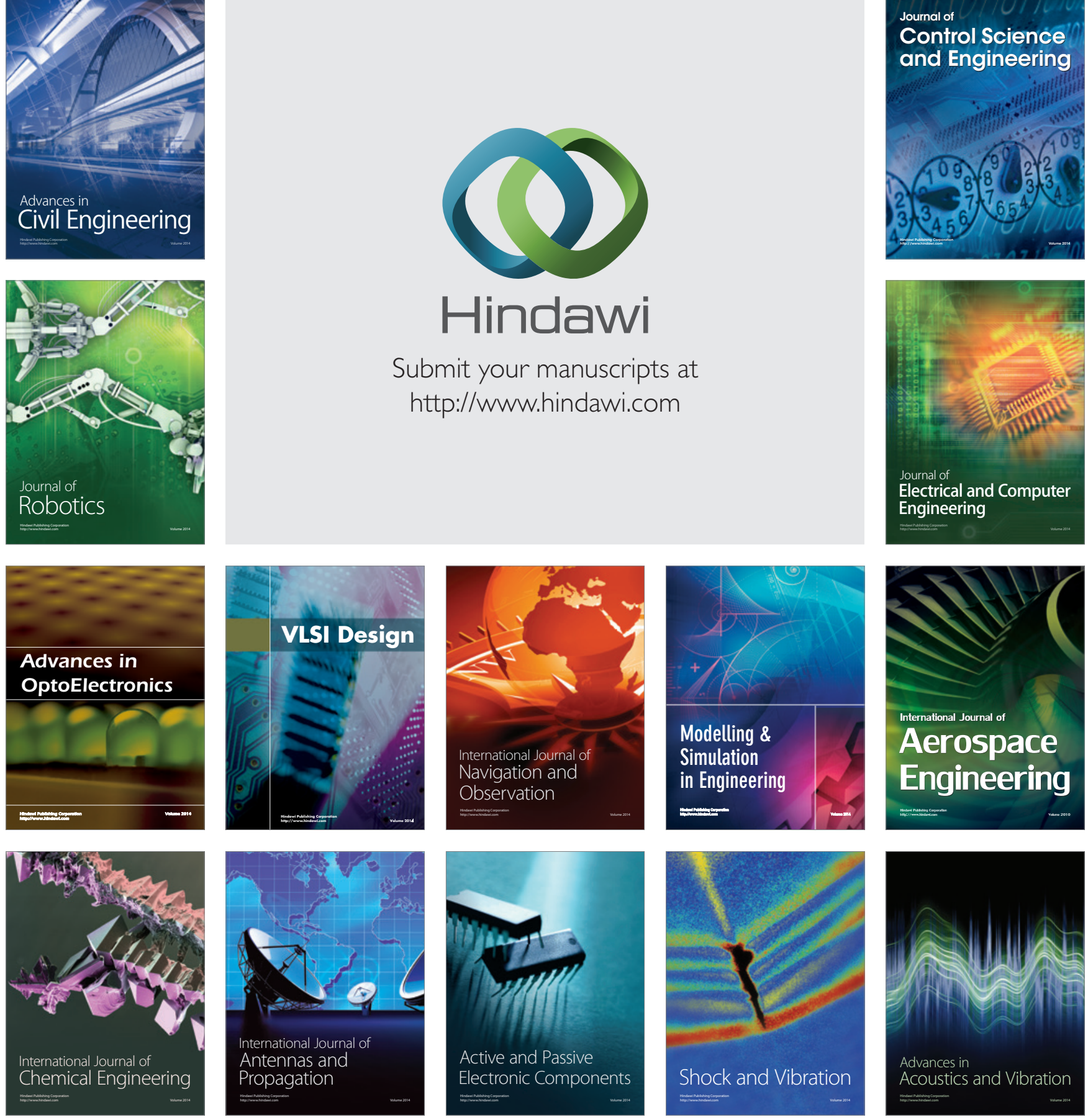